\title{
ON THE ASYMPTOTIC CHARACTER OF THE SOLUTIONS OF
}

\section{CERTAIN LINEAR DIFFERENTIAL EQUATIONS}

\section{CONTAINING A PARAMETER*}

BY

\section{GEORGE D. BIRKHOFF}

The aim of the present paper is to develop the asymptotic character of the solutions of linear differential equations of the form

$$
\frac{d^{n} z}{d x^{n}}+\rho a_{n-1}(x, \rho) \frac{d^{n-1} z}{d x^{n-1}}+\cdots+\rho^{n} a_{0}(x, \rho) z=0
$$

for large $|\rho|$. The functions $a_{i}(x, \rho)$ are analytic in the complex parameter $\rho$ at $\rho=\infty$ and have derivatives of all orders in the real variable $x$. Schlesinger $\dagger$ has proved asymptotic properties for $\lim \rho=\infty$ on some fixed $\operatorname{ray} \arg \rho=\alpha$; in this paper we prove similar properties for a region $\theta \leqq \arg \rho \leqq \psi$, but by a different method. In 1837 LiouviLLE $\ddagger$ treated the important special case

$$
\frac{d^{2} z}{d x^{2}}+\left[\rho^{2}+g(x)\right] z=0
$$

when $\rho$ is real, the first problem of the kind to be considered; the method of attack used in this paper is of a similar nature.

It is purposed to make an application of the results here obtained to boundary value and expansion problems in a second paper.

I desire to make acknowledgment of the kind encouragement and valuable suggestions received from Professor E. H. Moone, for both papers.

We consider functions $z(x, \rho)$ of a real variable $x$ on the closed interval

* Part of a paper presented to the Society (Chicago), Mar. 30, 1907, under a different title. Received for publication October 23, 1907.

† Matbematische Annalen, vol. 63 (1907), pp. 277-300. This paper appeared after the writing of the present paper. See also a paper by J. Horn, Mathematisohe Annalen, vol. 52 (1899), pp. 271-292.

$\ddagger$ Liouville's Journal, vol. 2, p. 16. Cf. in partioular 83. 
$(a, b)$ and of a complex parameter $\rho,|\rho|>R$, which satisfy a linear differential equation of the form

$$
\frac{d^{n} z}{d x^{n}}+\rho a_{n-1}(x, \rho) \frac{d^{n-1} z}{d x^{n-1}}+\cdots+\rho^{n} a_{0}(x, \rho) z=0 .
$$

Here we assume that the functions $a_{i}(x, \rho)$ are bounded by the inequalities

$$
\left|a_{i}(x, \rho)\right| \leqq M \quad(a \leqq x \leqq b,|\rho|>R),
$$

and that

$$
a_{i}(x, \rho)=\sum_{j=0}^{\infty} a_{i j}(x) \rho^{-j} .
$$

The coefficients $a_{i j}$ of this last series we assume to be continuous with continuous derivatives of all orders on $(a, b)$, but not necessarily real. As a consequence of (2) and (3) the $a_{i}(x, \rho)$ are continuous in $x$ and $\rho$, for the series (3) are series of continuous functions of $x$ and $\rho$ converging uniformly for $|\rho| \geqq R_{0}>R$. Further we postulate that the $n$ roots

of the equation

$$
w_{1}(x), w_{2}(x), \cdots, w_{n}(x)
$$

$$
w^{n}+a_{n-1,0}(x) w^{n-1}+\cdots+a_{00}(x)=0
$$

are distinct for every $x$ on $(a, b)$.

By $R(\mu)$ we denote the real part of the complex number $\mu$.

Definition. By a region $S$ of the p-plane we understand a region for which the indices 1 to $n$ can be so arranged that

$$
R\left[\rho w_{1}(x)\right] \leqq R\left[\rho w_{2}(x)\right] \leqq \ldots \leqq R\left[\rho w_{n}(x)\right]
$$

for every $x$ on $(a, b)$ and every $\rho$ on $S$.

If $\rho=\rho_{0}$ is an $S$ point, so that (5) obtains for $\rho=\rho_{0}$, it is clear that (5) holds if only $\arg \rho=\arg \rho_{0}$. By virtue of this fact the half line $\arg \rho=\arg \rho_{0}$ belongs to the same region $S$. For a given $x$ the relations (5) define a certain closed sector,

$$
\theta_{x} \leqq \arg \rho \leqq \psi_{x},
$$

containing the half line $\arg \rho=\arg \rho_{0}$. The largest closed sector common to all these sectors,

$$
\theta \leqq \arg \rho \leqq \psi,
$$

forms the region $S$, degenerating to a half line when $\theta=\psi$. The fundamental theorem refers to the solutions of (1) on these regions $S$, when such regions exist. 
An important set of inequalities on a region $S$ is

$$
\left|e^{p \int_{a}^{\beta} v_{1}(t) d t}\right| \leqq\left|e^{p \int_{a}^{\beta} w_{2}(t) d t}\right| \leqq \ldots \leqq\left|e^{p \int_{a}^{\beta} w_{k}(t) d t}\right| \quad(a \leqq a \leqq \beta \leqq b) .
$$

In an expression of the form

the term

$$
e^{\rho \theta(x)}\left[e_{0}(x) \rho^{-k}+e_{1}(x) \rho^{-k-1}+\cdots\right]
$$

$$
e_{0}(x) \rho^{-k}
$$

shall be called the principal term.

Finally we introduce the notation

so that (1) becomes

$$
\phi^{[k]}(x, \rho)=\rho^{-k} \frac{\partial^{k}}{\partial x^{k}} \phi(x, \rho)
$$

$$
A(z) \equiv z^{[n]}+a_{n-1}(x, \rho) z^{[n-1]}+\cdots+a_{0}(x, \rho) z=0 ;
$$

and also the notation $E$ as a generic notation for functions of $\rho$ and other variables bounded for $|\rho| \geqq R^{0}$, when $R^{0}$ is sufficiently large.

Lamas I. For every value of $i$ from 1 to $n$ inclusive there exist an infinite number of functions $u_{i 0}(x), u_{i 1}(x), \ldots$, continuous and with continuous derivatives of all orders, such that $u_{i 0}(x)$ does not vanish at any point of $(a, b)$ and such that if the functions

$$
u_{i}(x, \rho)=e^{\rho \int_{a}^{x} v_{i}(t) d t} \sum_{j=0}^{m-1} u_{i j}(x) \rho^{-j}
$$

be substituted for $z$ in the expression $A(z)$, the coefficient of

$$
e^{p \int_{a}^{x} w_{i}(t) d t} \rho^{-s}
$$$$
\left(\begin{array}{l}
i=1,2, \cdots, n \\
s=0,1, \cdots, m
\end{array}\right)
$$

in the expression thus obtained vanishes identically.

To prove this, write

$$
u(x, \rho)=e^{\rho \int_{a}^{x} v(t) d t} v(x, \rho),
$$

where $w(x)$ is some definite one of the $n$ roots $w_{i}(x)$. We find then $u^{[1]}(x, \rho) \equiv e^{\rho \int_{a}^{x} w(t) d t}\left[w(x) v(x, \rho)+v^{[1]}(x, \rho)\right]$, $u^{[2]}(x, \rho) \equiv e^{\rho \int_{a}^{x} v(t) d t}\left[\left([v(x)]^{2}+\frac{1}{\rho} \frac{d}{d x} v(x)\right) v(x, \rho)+2 v(x) v^{[1]}(x, \rho)+v^{[2]}(x, \rho)\right]$, and, in general,

$$
u^{[j]}(x, \rho)=e^{\rho \int_{a}^{x} w(t) d t} \sum_{k=0}^{j} \alpha_{j k}(x, \rho) v^{[k]}(x, \rho),
$$


in which $a_{j k}(x, \rho)$ is a polynomial in $1 / \rho$ of degree $j-1$ at most, whose coefficients are functions of $x$ continuous on $(a, b)$ and with continuous derivatives of all orders. We note that the principal term in the coefficient of $v(x, \rho)$ in (9) is

$$
\alpha_{j 00}(x)=[v(x)]^{j},
$$

and the principal term in the coefficient of $v^{[1]}(x, \rho)$ is

$$
\alpha_{j 10}(x)=j[w(x)]^{j-1} ;
$$

also we note that

Hence we conclude

$$
\alpha_{j j}(x, \rho)=1 \text {. }
$$

$$
A[u(x, \rho)] \equiv e^{\rho \int_{a}^{x} w(t) d t} A[v(x, \rho)]
$$

where

$$
\bar{A}(z) \equiv z^{[n]}+\bar{a}_{n-1}(x, \rho) z^{[n-1]}+\cdots+\bar{a}_{0}(x, \rho) z,
$$

the $\bar{a}_{j}(x, \rho)$ being conditioned as the $a_{j}(x, \rho)$ are in equations (2) and (3). For convenience we write

$$
\bar{a}_{n}(x, \rho)=1, \quad \bar{a}_{l}(x, \rho)=0
$$

We see from $(10),(11)$ that if we place

then

$$
\bar{a}_{l}(x, \rho) \equiv \sum_{j=0}^{\infty} \bar{a}_{\mathfrak{l}}(x) \rho^{-j},
$$

$$
\bar{a}_{00}(x)=[w(x)]^{n}+a_{n-1,0}(x)[w(x)]^{n-1}+\cdots+a_{00}(x)=0
$$

and

$$
\bar{a}_{10}(x)=n[10(x)]^{n-1}+(n-1) a_{n-1,0}(x)[w(x)]^{n-2}+\cdots+a_{10}(x),
$$

so that $\bar{a}_{10}(x) \neq 0$ on $(a, b)$, the $n$ roots $w_{i}(x)$ being distinct.

If then we write in (12)

$$
v(x, \rho)=\sum_{j=0}^{m-1} u_{j}(x) \rho^{-j},
$$

we find the condition that the coefficient of

in $A[u(x, \rho)]$ vanishes to be

$$
e^{p \int_{a}^{x} w(t) d t} \rho^{-\bullet}
$$$$
(s=0,1, \cdots, m)
$$

$$
\sum_{j+k+l=s} \bar{a}_{b j}(x) \frac{d^{l}}{d x^{l}} u_{k}(x)=0 .
$$

The equation (16) is true for $s=0$ by (14). For $s \neq 0$ we can write (16) in the form

$$
\bar{a}_{10}(x) \frac{d}{d x} u_{o-1}(x)+\bar{a}_{01}(x) u_{o-1}(x)+\sum_{j+k+l=s}^{k<s-1} \bar{a}_{y}(x) \frac{d^{l}}{d x^{l}} u_{k}(x)=0,
$$


since, if in (16) $k=s$, then $l=j=0$ and the term corresponding to this set of values has a coefficient $\bar{a}_{00}(x)=0$; if $k=8-1$, we have either $l=1$, $j=0$ or $l=0, j=1$, and the corresponding terms are the first terms of (17).

It appears then that $u_{d-1}(x)$ can be determined in terms of $u_{0-2}(x), u_{0-3}(x), \ldots, u_{0}(x)$ as a solution of a certain linear differential equar tion of the first order which has no singular points on $(a, b)$ since $\bar{a}_{10}(x) \neq 0$. Thus $u_{0}(x), u_{1}(x), \cdots, u_{m-1}(x)$ are obtained in succession from (16) for $s=1,2, \cdots, m$. For each $w_{i}(x)$ we obtain in this way a sequence of functions $u_{i 0}(x), u_{i 1}(x), \ldots$, such that if the expressions

$$
u_{i}(x, \rho)=e^{\rho \int_{a}^{x} w_{i}(t) d t} \sum_{j=0}^{m-1} u_{i j}(x) \rho^{-s}
$$

be substituted for $z$ in $A(z)$, the coefficient of

$$
e^{\rho \int_{a}^{x_{w}} w_{t}(t) d t} \rho^{-s} \quad(i=1,2, \cdots, n ; s=0,1, \cdots, m)
$$

vanishes by (17) since the conditions (16) are now satisfied for $s=1,2, \ldots, m$. Furthermore the differential equation for $u_{i 0}(x)$ is homogeneous, so that by taking for $u_{i 0}(x)$ a solution which is different from zero at one point of $(a, b)$, we are sure that $u_{i 0}(x)$ does not vanish at any point of $(a, b)$. Since the $a_{i j}(x)$ were continuous with all of their derivatives, the functions $u_{i 0}(x), u_{i 1}(x), \cdots$ are also continuous with all of their derivatives. The sequence of functions $u_{i 0}(x), u_{i 1}(x), \ldots$ has then the properties stated in the lemma.

If the formal developments

$$
e^{p \int_{a}^{x} w_{i}(t) d t} \sum_{j=0}^{\infty} u_{i j}(x) \rho^{-j}
$$$$
(i=1,2, \cdots, n)
$$

converged and admitted of $n$-fold term by term differentiation, we should have in them the asymptotic developments of solutions of our differential equation which we desire. This however will not in general be the case. We shall, nevertheless, show in the theorem below that by breaking off after $m$ terms of these series (i. e., by retaining the part $u_{i}(x, \rho)$ ) and putting in certain remainder terms we can get true solutions of our differential equation. In order to prove this we must first prove another lemma in which the form of the differential equation is established which is satisfied by the sums of these $m$ terms (i. e., $\left.u_{i}(x, \rho)\right)$ without any remainder :

LEMmA II. The homogeneous linear differential equation of order $n$ with $n$ solutions $u_{i}(x, \rho)$ has the form

$$
B(z) \equiv z^{[n]}+b_{n-1}(x, \rho) z^{[n-1]}+\cdots+b_{0}(x, \rho) z=0,
$$

where for $|\rho|>R^{0}$

$$
\left|b_{i}(x, \rho)\right| \leqq M^{0} ; \quad b_{i}(x, \rho)=\sum_{i=0}^{\infty} b_{i j}(x) \rho^{-j} \quad(i=0,1, \cdots, n-1) .
$$


The coefficients $b_{i j}(x)$ which appear here are continuous together with all their derivatives, and

$$
b_{i j}(x)=a_{i j}(x) \quad(i=0,1, \cdots, n-1 ; j=0,1, \cdots, m) .
$$

The homogeneous linear differential equation of order $n$ with solutions $u_{i}(x, \rho)$ is

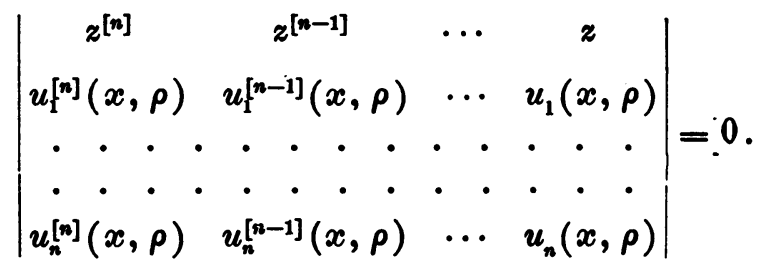

For the elements of this determinant we have

where by (10)

$$
u_{i}^{[j]}(x, \rho)=e^{\rho \int_{a}^{x} w_{i}(t) d t} \sum_{k=0}^{m+j-1} \lambda_{i j k}(x) \rho^{-k}
$$

Thus if we factor out of (20)

$$
\lambda_{i j 0}(x)=\left[w_{i}(x)\right]^{j} u_{i 0}(x) .
$$

$$
\prod_{i=1}^{n} e^{p \int_{a}^{x} w_{i}(t) d t}
$$

the differential equation takes the form

$$
\beta_{n}(x, \rho) z^{[n]}+\beta_{n-1}(x, \rho) z^{[n-1]}+\cdots+\beta_{0}(x, \rho) z=0,
$$

where $\beta_{i}(x, \rho)$ are polynomials in $1 / \rho$. We have for the principal term of $\beta_{n}(x, \rho)$,

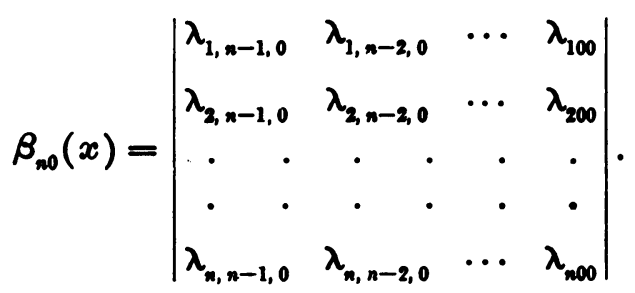

In view of (22) this last determinant may be written

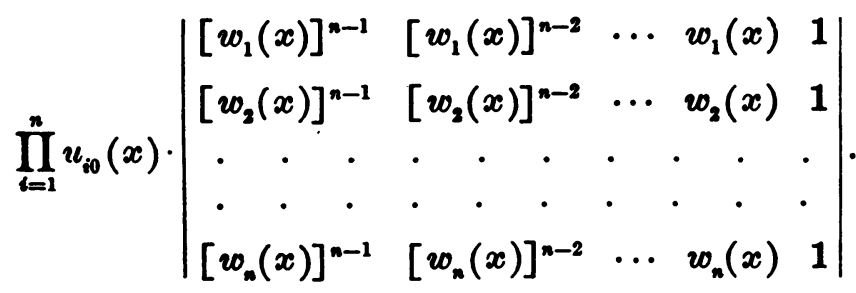


This is not zero at any point on $(a, b)$ as the $w_{i}(x)$ are distinct and the $u_{i 0}(x) \neq 0$ by Lemma I. Therefore for $|\rho| \geqq R^{0}$

$$
\left|\frac{\beta_{i}(x, \rho)}{\beta_{n}(x, \rho)}\right| \leqq M^{0} ; \quad b_{i}(x, \rho)=\frac{\beta_{i}(x, \rho)}{\beta_{n}(x, \rho)}=\sum_{j=0}^{\infty} b_{i j}(x) \rho^{-j} .
$$

But we can write (23) in the form

$$
z^{[n]}+b_{n-1}(x, \rho) z^{[n-1]}+\cdots+b_{0}(x, \rho) z=0 .
$$

Now the functions $b_{i}(x)$ and their derivatives are continuous since the $w_{i}(x)$ and $u_{i}(x)$ are of this character. The first part of the lemma is thus proved.

Now let $j_{0}$ be the smallest value of $j$ for which, for some $i$ and $x$,

$$
b_{i j}(x) \neq a_{i j}(x) \text {. }
$$

From (21) and (22) we see that the principal term of

$$
B\left[u_{i}(x, \rho)\right]-A\left[u_{i}(x, \rho)\right] \equiv \overline{B-A}\left[u_{i}(x, \rho)\right]
$$

is

$$
\rho^{-j_{0}}\left[\sum_{k=0}^{n-1}\left[b_{k j_{0}}(x)-a_{k j_{0}}(x)\right]\left[w_{i}(x)\right]^{k}\right] u_{i 0}(x) .
$$

Assume $j_{0} \leqq m$ if possible. In each part of the difference (25) the coefficient of

$$
e^{p \int_{a}^{x} v_{i}(t) d t} \rho^{-j_{0}}
$$

must then vanish, in the first part since $u_{i}(x, \rho)$ are solutions of $B(z)=0$; in the second part, by Lemma I. Therefore from (26)

We conclude that

$$
\sum_{k=0}^{n-1}\left[b_{k j_{0}}(x)-a_{k j_{0}}(x)\right]\left[w_{i}(x)\right]^{k}=0 \quad(i=1,2, \cdots, n) .
$$

$$
b_{k j_{0}}(x)=a_{k j_{0}}(x) \quad(k=0,1, \cdots, n-1),
$$

the $v_{i}(x)$ being distinct. This is a contradiction. Hence $j_{0}>m$.

Theorem. On a region $S$ there exist $n$ independent solutions

of

$$
z_{1}(x, \rho), z_{2}(x, \rho), \cdots, z_{n}(x, \rho)
$$

$$
\frac{d^{n} z}{d x^{n}}+\rho a_{n-1}(x, \rho) \frac{d^{n-1} z}{d x^{n-1}}+\cdots+\rho^{n} a_{0}(x, \rho) z=0
$$

analytic in $\rho$ such that if the integer $m$ is chosen at pleasure and $\rho$ is on $S$,

$$
z_{i}(x, \rho)=u_{i}(x, \rho)+e^{\rho \int_{a}^{x_{w}} w_{i}(t) d t} \cdot E_{0} \rho^{-m},
$$




$$
\begin{aligned}
& \frac{d}{d x} z_{i}(x, \rho)=\frac{d}{d x} u_{i}(x, \rho)+e^{\rho \int_{a}^{x} w_{i}(t) d t} \cdot E_{1} \rho^{-m+1}, \\
& \frac{d^{n-1}}{d x^{n-1}} z_{i}(x, \rho)=\frac{d^{n-1}}{d x^{n-1}} u_{i}(x, \rho)+e^{\rho \int_{a}^{x} w_{i}(t) d t} \cdot E_{n-1} \rho^{-m+n-1},
\end{aligned}
$$

where

$$
u_{i}(x, \rho)=e^{\rho \int_{a}^{x} w_{i}(t) d t} \sum_{j=0}^{m-1} u_{i j}(x) \rho^{-j}
$$

and $u_{i 0}(x)$ does not vanish at any point of $(a, b)$.

Proof. We proceed to effect a comparison of the solutions of the given differential equation with the solutions of the differential equation of Lemma II. First we write the given differential equation in the form

$$
\frac{d^{n} z}{d x^{n}}+\rho b_{n-1}(x, \rho) \frac{d^{n-1} z}{d x^{n-1}}+\cdots+\rho^{n} b_{0}(x, \rho) z=\rho^{n} \overline{B-A}(z) .
$$

The development of the coefficient of $z^{[k]}$ in $\overline{B-A}(z)$ begins with a term in $\rho^{-m-1}$, in view of $(18)$ and $(19)$, so that

$$
\left|b_{k}(x, \rho)-a_{k}(x, \rho)\right| \leqq D \cdot|\rho|^{-m-1}
$$

for $|\rho| \geqq R^{0}$.

The general solution of a non-homogeneous linear differential equation

$$
\frac{d^{n} y}{d x^{n}}+k_{n-1}(x) \frac{d^{n-1} y}{d x^{n-1}}+\cdots+k_{0}(x) y=\phi(x)
$$

may be written* in terms of a set of linearly independent solutions $y_{1}(x), y_{2}(x), \cdots, y_{n}(x)$ of the reduced equation in the form

$$
y=\sum_{i=1}^{n} c_{i} y_{i}(x)+\int_{a}^{x}\left[\sum_{i=1}^{n} y_{i}(x) z_{i}(\xi)\right] \phi(\xi) d \xi,
$$

where the functions $z_{1}(x), z_{2}(x), \cdots, z_{n}(x)$ are determined from the conditions

$$
\sum_{i=1}^{n}\left(\frac{d^{l}}{d x^{l}} y_{i}(x)\right) z_{i}(x)= \begin{cases}0 & (l=0,1, \cdots, n-2), \\ 1 & (l=n-1),\end{cases}
$$

while $c_{1}, c_{2}, \cdots, c_{n}$ are arbitrary constants. Any $y$ and $\phi$ satisfying (30) also satisfy (31) for some choice of $c_{1}, c_{2}, \ldots, c_{n}$; and if $y$ and $\phi$ satisfy (31) for some $c_{1}, c_{2}, \cdots, c_{n}$, they also satisfy (30); hence (30) and (31) are ontirely equivalent.

* CL., for inetance, SchuresivarkB : Hanulbweh der Theorie der livearen Differentialgleichungen, vol. 1, p. 78. 
Thus treating (28) as a non-homogeneous equation we find an equivalent equation

$$
z(x, \rho)=\sum_{i=1}^{n} c_{i} u_{i}(x, \rho)+\int_{a}^{x}\left[\sum_{i=1}^{n} u_{i}(x, \rho) v_{i}(\xi, \rho)\right] \rho^{n} \overline{B-A}[z(\xi, \rho)] d \xi
$$

where the $v_{i}$ 's are obtained from the equations

$$
\sum_{i=1}^{n} u_{i}^{[l]}(x, \rho) v_{i}(x, \rho)= \begin{cases}0 & (l=0,1, \cdots, n-2), \\ \rho^{-n+1} & (l=n-1) .\end{cases}
$$

If in place of these functions $v_{i}$ we introduce functions $\bar{u}_{i}$ defined by the equation

equation (32) becomes

$$
v_{i}(x, \rho)=\rho^{-n+1} \bar{u}_{i}(x, \rho),
$$

$$
z(x, \rho)=\sum_{i=1}^{n} c_{i} u_{i}(x, \rho)+\rho \int_{a}^{x}\left[\sum_{i=1}^{n} u_{i}(x, \rho) \bar{u}_{i}(\xi, \rho)\right] \overline{B-A}[z(\xi, \rho)] d \xi,
$$

where the $\bar{u}_{i}$ are determined from the equations

$$
\sum_{i=1}^{n} u_{i}^{[l]}(x, \rho) \bar{u}_{i}(x, \rho)= \begin{cases}0 & (l=0,1, \cdots, n-2), \\ 1 & (l=n-1) .\end{cases}
$$

In order to prove the existence of a solution $z_{k}(x, \rho)$ of the character stated in the theorem for some definite $k$ we make the final transformation of the constants $c_{i}$ in (33),

$$
\begin{array}{rr}
c_{i}=c_{i}^{\prime} & (i=1,2, \cdots, k), \\
c_{i}=-\rho \int_{a}^{b} \bar{u}_{i}(\xi, \rho) \overline{B-A}[z(\xi, \rho)] d \xi+c_{i}^{\prime} & (i=k+1, k+2, \cdots, n) .
\end{array}
$$

This transformation is reversible. The given differential equation thus appears finally in the form of an equivalent integral equation

$$
\begin{aligned}
z(x, \rho)=\sum_{i=1}^{n} c_{i}^{\prime} u_{i}(x, \rho) & +\rho \int_{a}^{x}\left[\sum_{i=1}^{k} u_{i}(x, \rho) \bar{u}_{i}(\xi, \rho)\right] \overline{B-A}[z(\xi, \rho)] d \xi \\
& +\rho \int_{b}^{x}\left[\sum_{i=k+1}^{n} u_{i}(x, \rho) \bar{u}_{i}(\xi, \rho)\right] \overline{B-A}[z(\xi, \rho)] d \xi,
\end{aligned}
$$

that is, the solutions of (36) and $A(z)=0$ are the same. In the form (33) we could infer one and but one solution $z(x, \rho)$ for a given set of $c_{1}, c_{2}, \cdots, c_{n}$; in fact, that solution of $A(z)=0$ which satisfies the conditions

$$
\frac{d^{j}}{d x^{j}} z(a, \rho)=\sum_{i=1}^{n} c_{i} \frac{d^{j}}{d x^{j}} u_{i}(a, \rho) \quad(j=0,1, \cdots, n-1),
$$

but a similar inference is not possible for (36). 
Write

$$
\begin{aligned}
& \sum_{i=1}^{k} u_{i}^{[l]}(x, \rho) \bar{u}_{i}(\xi, \rho)=e^{\rho \int_{\xi}^{x} w_{k}(t) d t} \phi_{k l}(x, \xi ; \rho), \\
& \sum_{i=k+1}^{n} u_{i}^{[l]}(x, \rho) \bar{u}_{i}(\xi, \rho)=e^{\rho \int_{\xi}^{x} w_{k}(t) d t} \Psi_{k l}(x, \xi ; \rho) .
\end{aligned}
$$

For the consideration of (36) it is fundamental to establish that the functions $\phi_{k l}$ and $\psi_{k l}$ are bounded in the following manner for $|\rho| \geqq R^{0}$ :

$$
\begin{array}{ll}
\left|\phi_{k l}(x, \xi ; \rho)\right| \leqq T, & a \leqq \xi \leqq x \leqq b \\
\left|\psi_{k l}(x, \xi ; \rho)\right| \leqq T, & a \leqq x \leqq \xi \leqq b
\end{array}
$$

To prove this we note first that from (21)

$$
u_{i}^{[l]}(x, \rho)=e^{\rho \int_{a}^{x} w_{i}(t) d t} \eta_{i l}(x, \rho),
$$

in which $\eta_{i l}(x, \rho)$ denotes a polynomial in $1 / \rho$. If now we substitute in (34)

$$
\bar{u}_{i}(x, \rho)=e^{-\rho \int_{a}^{x} w_{i}(t) d t} \bar{\eta}_{i}(x, \rho),
$$

we find for the determination of $\bar{\eta}_{i}(x, \rho)$ the $n$ linear equations

$$
\sum_{i=1}^{n} \eta_{i i}(x, \rho) \bar{\eta}_{i}(x, \rho)= \begin{cases}0 & (l=0,1, \cdots, n-2), \\ 1 & (l=n-1) .\end{cases}
$$

The determinant of these equations is a polynomial in $1 / \rho$ which we called $\beta_{n}(x, \rho)$, and has the principal term $\beta_{n 0}(x)$ [see (23)] which is not zero. We conclude that for $|\rho| \geqq R^{0}$

$$
\left|\eta_{i l}(x, \rho)\right| \leqq \eta, \quad\left|\bar{\eta}_{i}(x, \rho)\right| \leqq \eta .
$$

Now from (39) and (40) we have

$$
\left|u_{i}^{[l]}(x, \rho) \bar{u}_{i}(\xi, \rho)\right| \leqq\left|e^{\rho / \int_{\xi}^{x} w_{i}(t) d t} \eta^{2}\right|,
$$

whence

$$
\left|u_{i}^{[l]}(x, \rho) \bar{u}_{i}(\xi, \rho)\right| \leqq\left|e^{\rho / \int_{\xi}^{x} w_{k}(i) d t} \eta^{2}\right|,
$$

if we make the restriction

$$
\xi \leqq x \quad(i=1,2, \cdots, k), \quad \xi \geqq x \quad(i=k+1, k+2, \cdots, n) .
$$

To see this one recalls the inequalities (7). Hence

$$
\begin{aligned}
\left|\sum_{i=1}^{k} u_{i}^{[l]}(x, \rho) \bar{u}_{i}(\xi, \rho)\right| \leqq k\left|e^{\rho \int_{\xi}^{x} w_{k}(t) d t} \eta^{2}\right| & (\xi \leqq x), \\
\left|\sum_{i=k+1}^{n} u_{i}^{[l]}(x, \rho) \bar{u}_{i}(\xi, \rho)\right| \leqq(n-k)\left|e^{\rho / \int_{\xi}^{x} w_{k}(t) d t} \eta^{2}\right| & (\xi \geqq x),
\end{aligned}
$$

which are in effect inequalities (38). 
We now consider that solution $z_{k}(x, \rho)$ of $(36)$ for which

and we will show

$$
c_{i}^{\prime}= \begin{cases}0 & (i \neq k), \\ 1 & (i=k),\end{cases}
$$

(a) that for $|\rho| \geqq R^{\prime}$ one and but one such solution exists, and this is analytic in $\rho$;

(b) that the solutions $z_{1}, z_{2}, \ldots, z_{n}$ thus defined fulfill the relations (27) of our fundamental theorem.

The linear independence of $z_{1}, z_{2}, \ldots, z_{n}$ is then an immediate consequence of their form (27), since $u_{1}, u_{2}, \ldots, u_{n}$ are linearly independent. The demonstration is thus completed.

Proof of $(a)$.

We know there exists one and but one solution of (33) for all sets of values of $c_{i}$. To each set corresponds a definite transformed set $c_{i}^{\prime}$ which we will show has the form

$$
c_{i}^{\prime}=\sum_{j=1}^{n} \gamma_{i j}(\rho) c_{j}
$$

where the $\gamma_{i j}(\rho)$ are analytic in $\rho$. To prove this statement we define $Z_{k}(x, \rho),(k=1,2, \cdots, n)$, to be that solution of $A(z)=0$ which satisfies the equations

$$
\frac{d^{j}}{d x^{j}} Z_{k}(a, \rho)=\frac{d^{j}}{d x^{j}} u_{k}(a, \rho) \quad(j=0,1, \cdots, n-1) .
$$

If $z$ is the solution of (32) for the set $c_{1}, c_{2}, \cdots, c_{n}$, we have then

$$
z=\sum_{j=1}^{n} c_{j} Z_{j}(x, \rho) \text {. }
$$

The $Z_{k}(x, \rho)$ are analytic in $\rho$ since the coefficients in $A(z)=0$ and the $u_{i}(x, \rho)$ are. If this value of $z$ is substituted in (35), we obtain the transformation in the stated form.

From this we see that either a unique solution for the set $c_{i}^{\prime}$ of (46) exists, in which case this solution will be analytic in $\rho$, or there is a solution of (36) for $c_{i}^{\prime}=0(i=1,2, \cdots, n)$. If then we prove that the latter alternative is impossible for $|\rho|>R^{\prime}$, we shall have proved statement $(a)$.

Let us now write down (36) and the equations obtained from it by differentiation, using the definitions (37). We then obtain

$$
\begin{aligned}
& z^{[l]}(x, \rho)=\sum_{i=1}^{n} c_{i}^{\prime} \cdot u\left[_{i}^{[l]}(x, \rho)+\rho \int_{a}^{x} e^{\rho \int_{\xi}^{x} w_{k}(t) d t} \phi_{k k}(x, \xi ; \rho) \overline{B-A}[z(\xi, \rho)] d \xi\right. \\
& +\rho \int_{b}^{x} e^{\rho / \int_{\xi}^{x} w_{k}(t) d t} \psi_{k l}(x, \xi ; \rho) \overline{B-A}[z(\xi, \rho)] d \xi \\
& (l=0,1, \cdots, n-1) \text {. }
\end{aligned}
$$


These are a set of linear integral equations in $z(x, \rho), z^{[1]}(x, \rho), \ldots, z^{[n-1]}(x, \rho)$ of the Fredholm type.

Assume if possible a solution $z(x, \rho)$ to exist for $c_{i}^{\prime}=0(i=1,2, \cdots, n)$. If we write

$$
z^{[l]}(x, \rho)=e^{\rho \int_{a}^{x} w_{k}(t) d t} z_{l}(x, \rho) \quad(l=0,1, \cdots, n-1),
$$

the equations (47) become

$$
\begin{aligned}
& x_{l}(x, \rho)=\rho \int_{a}^{x} \phi_{k l}(x, \xi ; \rho) g[z(\xi, \rho)] d \xi+\rho \int_{b}^{x} \psi_{k l}(x, \xi ; \rho) g[z(\xi, \rho)] d \xi \\
& (l=0,1, \cdots, n-1)
\end{aligned}
$$

where

$$
g[z(x, \rho)] \equiv \sum_{j=0}^{n-1}\left[b_{j}(x, \rho)-a_{j}(x, \rho)\right] z_{j}(x, \rho) .
$$

If $W \neq 0$ be the maximum of

$$
\left|z_{l}(x, \rho)\right| \quad(l=0,1, \cdots, n-1)
$$

on $(a, b)$, we conclude from (29) that for $|\rho| \geqq R^{0}$

$$
|g[z(\xi, \rho)]| \leqq n D \cdot W \cdot|\rho|^{-m-1} \text {. }
$$

But in one of the equations (48), e. g., $l=l_{1},\left|z_{l}(x, \rho)\right| s$ the value $W$ at $x=x_{1}$, so that

$$
W=\left|\rho \int_{a}^{x_{1}} \phi_{k l_{1}}\left(x_{1}, \xi ; \rho\right) g[z(\xi, \rho)] d \xi+\rho \int_{b}^{x_{1}} \psi_{k l_{1}}\left(x_{1}, \xi ; \rho\right) g[z(\xi, \rho)] d \xi\right| .
$$

Applying to (51) inequalities (50) and (38), as is possible $s^{i}$ ce $\xi \leqq x_{1}$ in $\phi_{k l_{1}}\left(x_{1}, \xi ; \rho\right)$ of $(38) \xi \geqq x_{1}$ and in $\psi_{k l_{1}}\left(x_{1}, \xi ; \rho\right)$, we find

$$
W \leqq n(b-a) T \cdot D \cdot W \cdot|\rho|^{-m}
$$

which is not possible for

$$
|\rho| \geqq \sqrt[m]{n(b-a) T \cdot D} .
$$

Thus the set of values $c_{i}^{\prime}=0$ is seen to be impossible for $|\rho| \geqq R^{\prime}$. Hence a unique solution for (46) exists.

Proof of $(b)$.

By (46) we have

$$
\begin{array}{r}
z_{k}^{[l]}(x, \rho)=u_{k}^{[l]}(x, \rho) \\
+\rho \int_{a}^{x}\left[\sum_{i=1}^{k} u_{i}^{[l]}(x, \rho) \bar{u}_{i}(\xi, \rho)\right] \overline{B-A}\left[z_{k}(\xi, \rho)\right] d \xi \\
+\rho \int_{b}^{x}\left[\sum_{i=k+1}^{n} u_{i}^{[l]}(x, \rho) \bar{u}_{i}(\xi, \rho)\right] \overline{B-A}\left[z_{k}(\xi, \rho)\right] d \xi .
\end{array}
$$

Writing then

$$
x_{b}^{[l]}(x, \rho)=e^{p \int_{a}^{x} w_{k}(t) d t} z_{m l}(x, \rho) \text {, }
$$


we obtain

$$
\begin{aligned}
& z_{k l}(x, \rho)=\eta_{k l}(x, \rho)+\rho \int_{a}^{x} \phi_{k l}(x, \xi ; \rho) g\left[z_{k}(\xi, \rho)\right] d \xi \\
&+\rho \int_{b}^{x} \psi_{k l}(x, \xi ; \rho) g\left[z_{k}(\xi, \rho)\right] d \xi,
\end{aligned}
$$

where $\eta_{k l}(x, \rho)$ and $g(z)$ are defined as in (39) and (49). This equation (54) is analogous to (48).

Let $W$ denote the maximum of

$$
\left|z_{k l}(x, \rho)\right| \quad(l=0,1, \cdots, n-1)
$$

on $(a, b)$. Then we find in analogy with (50) that for $|\rho| \geqq R^{0}$

$$
\left|g\left[z_{k}(x, \rho)\right]\right| \leqq n D \cdot W \cdot|\rho|^{-m-1} \text {. }
$$

If this maximum be attained for $l=l_{1}, x=x_{1}$, we find in analogy with (52)

$$
W \leqq\left|\eta_{k l_{1}}\left(x_{1}, \rho\right)\right|+n(b-a) T \cdot D \cdot W|\rho|^{-m},
$$

whence, if $m>0$ and $|\rho|$ is large,

$$
W \leqq Q
$$

since

But from (54)

$$
\left|\eta_{k l_{1}}\left(x_{1}, \rho\right)\right| \leqq \eta
$$

$$
\begin{aligned}
\left|z_{k l}(x, \rho)-\eta_{k l}(x, \rho)\right|=\mid \rho \int_{a}^{x} \phi_{k l}(x, \xi ; \rho) g\left[z_{k}(\xi, \rho)\right] d \xi & \\
& +\rho \int_{b}^{x} \psi_{k l}(x, \xi ; \rho) g\left[z_{k}(\xi, \rho)\right] d \xi \mid .
\end{aligned}
$$

Therefore, using (38) and the inequality

$$
\left|g\left[z_{k}(x, \rho)\right]\right| \leqq n D \cdot Q|\rho|^{-m-1},
$$

which is a consequence of (56), we see that for a large enough $|\rho|$

$$
\left|z_{k l}(x, \rho)-\eta_{k l}(x, \rho)\right| \leqq n(b-a) T \cdot D \cdot Q|\rho|^{-m} \text {. }
$$

Recalling that $E$ is a generic notation for functions of $\rho$ and other variables bounded for large $|\rho|$, we conclude from this at once that

$$
\begin{aligned}
& z_{k}(x, \rho)=u_{k}(x, \rho)+e^{\rho \int_{a}^{x} w_{k}(t) d t} E_{0} \cdot \rho^{-m}, \\
& \frac{d}{d x} z_{k}(x, \rho)=\frac{d}{d x} u_{k}(x, \rho)+e^{\rho \int_{a}^{x} w_{k}(t) d t} E_{1} \cdot \rho^{-m+1}, \\
& \frac{d^{n-1}}{d x^{n-1}} z_{k}(x, \rho)=\frac{d^{n-1}}{d x x^{n-1}} u_{k}(x, \rho)+\rho^{\rho \int_{a}^{x} v_{k}(t) d t} E_{n-1} \cdot \rho^{-m+n-1}
\end{aligned}
$$

